\title{
Using Health Care Resources Wisely During and Following the COVID-19 Pandemic
}

\author{
Karen B. Born, Wendy Levinson
}

The declaration of COVID-19 as a global pandemic was met with a call for Canadians to stay home as workplaces, schools, businesses, and community spaces shuttered. Yet, the doors of the health care system have remained open and individual providers, hospitals, and organizations rapidly adapted to care for Canadians in this complex new environment. Clinicians and providers have encountered and managed a myriad of challenges, including initial shortages of personal protective equipment, surges of COVID-19 patient care demands, a rapid shift to virtual care, and a depleted health care workforce. In the pandemic era, the Choosing Wisely Canada campaign has taken on new meanings and urgency. ${ }^{1}$

Patient expectations of care and health care utilization patterns also rapidly changed in response to the pandemic. A systematic review on the impact of the pandemic on health services utilization in mainly high-income countries found that utilization decreased by more than one-third in the early months of the pandemic. ${ }^{2}$ The authors overlay these findings with existing evidence that up to one-third of health care services offer no clinical value to patients. ${ }^{3}$ This has been supported by Canadian data as well. ${ }^{4}$ For example, March and April 2020 saw a 50\% decline in emergency department visits in Canada. Similarly, hospital admissions decreased in this period by $36 \%$, driven in part by an almost $50 \%$ decline in surgical procedures. ${ }^{5}$

There are clearly drops in utilization for necessary health care services and procedures during the pandemic; however, there is growing literature suggesting that some decreases in utilization have been driven by declines in overused and low-value tests and treatments. ${ }^{6}$ As resources have shifted to pandemic priorities and essential care needs, these unnecessary tests and treatments have also declined. This may be due in part to rapid shifts to virtual care, as well as patients and providers weighing the costs of contact. The pandemic added a layer of risk consideration of contracting COVID-19 during medical appointments, procedures, or tests, as well as concerns about consuming scarce personal protective equipment. ${ }^{7}$

When the pandemic recedes, it will be imperative to rebuild and meet the challenges ahead. In the post-pandemic era, and with its anticipated financial constraints, health care systems may need to continue to do more with less. There is an anticipated surge of patient demands including addressing backlogs of deferred and delayed care such as surgeries. ${ }^{8}$ Further, the pandemic is both indirectly and directly driving population health needs for mental health services as well as chronic disease management. Sustained reductions in low-value tests and treatments can help meet these needs.

Choosing Wisely Canada and CADTH are proactively working to highlight key recommendations from evidence-based lists of recommendations to inform priorities for rebuilding, which include avoiding low-value care. Rebuilding the health care system in the post-pandemic era needs to take into account a diversity of perspectives on how to prioritize high-value care for those who need it most, including clinician, patient, and policy expert perspectives. 


\section{References}

1. Soong C, Born KB, Levinson W. Less is more, now more than ever. BMJ Quality \& Safety. 2021;30:56-58. doi: 10.1136/ bmjqs-2020-011444

2. Moynihan R, Sanders S, Michaleff ZA, et al. Impact of COVID-19 pandemic on utilisation of healthcare services: a systematic review. BMJ Open. 2021;11:e045343. doi: 10.1136/bmjopen-2020-045343

3. Canadian Institute for Health Information. Unnecessary Care in Canada. Ottawa, ON: ClHI; 2017: https://www.cihi.ca/ sites/default/files/document/choosing-wisely-baseline-report-en-web.pdf

4. COVID-19's effect on hospital care services. Ottawa (ON): Canadian Institute for Health Information; November 2020: https://www.cihi.ca/en/covid-19-resources/impact-of-covid-19-on-canadas-health-care-systems/covid-19s-effect-onhospital. Accessed 2021 Apr 28.

5. How COVID-19 affected emergency departments. Ottawa (ON): Canadian Institute for Health Information; November 2020: https://www.cihi.ca/en/covid-19-resources/impact-of-covid-19-on-canadas-health-care-systems/how-covid-19affected. Accessed 2021 Apr 28.

6. Moynihan R, Johansson M, Maybee A, et al. Covid-19: an opportunity to reduce unnecessary healthcare BMJ 2020; 370 :m2752 doi: 10.1136/bmj.m2752

7. Bhatia RS, Shojania KG, Levinson W. Cost of contact: redesigning healthcare in the age of COVID. BMJ Quality \& Safety 2021;30:236-239. doi: 10.1136/bmjqs-2020-011624

8. Wang, J, Vahid, S, Eberg, M et al. Clearing the surgical backlog caused by COVID-19 in Ontario: a time series modelling study. CMAJ. Nov 2020;192(44):E1347-E1356. doi: 10.1503/cmaj.201521

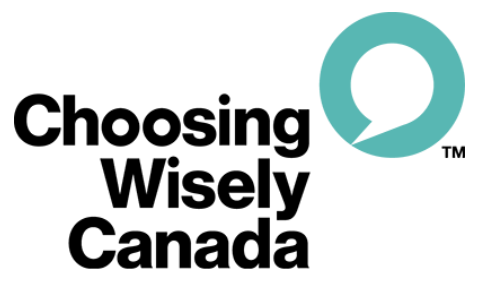

Choosing Wisely Canada is the national voice on health care overuse in Canada. Dr. Wendy Levinson is chair, Choosing Wisely Canada, and Professor of Medicine, University of Toronto. Karen B. Born, PhD, is knowledge translation lead, Choosing Wisely Canada, and Assistant Professor, Institute of Health Policy, Management and Evaluation, University of Toronto. 